\title{
LOS MODELOS DIMENSIONALES DE LA PERSONALIDAD Y SU IMPORTANCIA EN LA PSICOLOGÍA DE LA SALUD
}

\section{Dimensional Models of Personality and its Importance in Health Psychology}

\author{
Mariantonia Lemos ${ }^{1}$
}

doi: https://doi.org/10.17533/udea.rp.e342593

\section{Resumen}

La psicología de la salud es un campo de aplicación de la psicología en el que el estudio de los factores de riesgo y protección de enfermedades crónicas tiene una alta importancia. En este campo los estudios seńalan la importancia de la personalidad como un factor de vulnerabilidad para el desarrollo y el mal pronóstico de diferentes enfermedades crónicas. Esta revisión de la literatura presenta algunos de los modelos dimensionales de la personalidad y su relación con la salud/enfermedad, haciendo hincapié en el efecto de ciertos rasgos de personalidad en la enfermedad cardiovascular.

\section{Abstract}

Health psychology is an application field of psychology that studies risk and protective factors related to chronical illness. Different studies concluded the importance of personality as a vulnerability factor in clinical outcomes related to chronical illness. This theoretical review presents some of the dimensional models of personality and its relation with health-disease, emphasizing in some traits of personality and cardiovascular disease. It is discussed the reliability of con-

$$
\text { Recibido: 2020-06-22 / Aceptado: 2020-12-18 }
$$

Para citar este artículo en APA: Lemos, M. (2020).

Los modelos dimensionales de la personalidad y su importancia en la psicología de la salud. Revista de Psicologia Universidad de Antioquia, 12(2), e342593. doi: https://doi.org/10.17533/udea. rp.e342593
Se discute la validez de conceptos como el de personalidad tipo D, la relación de los modelos dimensionales con los temperamentales y se enfatiza en la importancia del modelo de cinco factores, específicamente del neuroticismo y de la extraversión, como factores de alta relevancia para la psicología de la salud.

Palabras clave: personalidad, cinco grandes factores de la personalidad, rasgos de personalidad, psicología de la salud, enfermedad cardiovascular.

cepts as type D personality, the relation of dimensional models with temperamental models and the importance of the five-factor personality model, in particular, neuroticism and extraversion as a highly relevant factors for health psychology.

Keywords: personality, five-factor personality model, personality traits, health psychology, cardiovascular disease.

1 Doctora en Psicología. Profesora Departamento de Psicología, Universidad Eafit. Correo: mlemosh@eafit.edu.co; http://orcid.org/00000002-9131-4704. 


\section{Introducción}

La psicología de la salud hace referencia a la aplicación de diversas contribuciones científicas y profesionales de la psicología al entendimiento e intervención en temas de salud, ya sea a nivel de la promoción de estilos de vida saludables, prevención de enfermedades, tratamiento de patologías específicas y evaluación del sistema sanitario (García et al., 2008; Kazarian y Evans, 2001). De esta manera el trabajo a realizar puede llevarse a cabo desde diferentes niveles como la asesoría individual y los talleres grupales, pero también el diseño de políticas públicas que afectan a una comunidad en general. No obstante, cualquier profesional de las ciencias sociales y humanas reconoce la importancia de las diferencias individuales, ya que afectan hasta qué punto los programas de promoción y prevención, o las decisiones que se toman frente a la salud, a pesar de las políticas que existan, se asocian con la conservación de la salud o llevan al desarrollo de la enfermedad.

Adicionalmente, estudios sobre el estrés han señalado cómo este proceso está influenciado por variables de orden individual como la personalidad, anotando que esta, la historia de aprendizaje y los recursos disponibles llevarían a la valoración de los estresores como potencial daño o pérdida, reto o amenaza (Everly y Lating, 2002; Lazarus, 1999). Así mismo, los estudios sobre factores de riesgo para la incidencia o el pronóstico de una enfermedad crónica - como la enfermedad cardiovascular, el cáncer o la artritis reumatoidehan evidenciado la importancia de variables emocionales como la depresión, ansiedad, episodios de ira, entre otros (Chida y Steptoe, 2009; Chimenti et al., 2019; Graham-Engeland et al., 2018; Islami et al., 2018; Lichtman et al., 2014; Moser et al., 2011; Rice et al., 2020). No obstante, también se ha reconocido la existencia de rasgos y tipos de personalidad que afectan el proceso, siendo la personalidad tipo A una de las primeras descritas en el campo de la psicología de la salud (Myrtek, 2001).

La personalidad o el patrón de conducta tipo a fue descrita por los cardiólogos Friedman y Roseman en los años 50 al referirse a un patrón que consistía en una personalidad competitiva, ansiosa en relación al tiempo, hostil y de una exagerada necesidad de logro (Friedman y Roseman, 1976). Durante 
varias décadas diversos estudios mostraron a este patrón conductual como un predictor independiente de la mortalidad cardiovascular, y hasta se llevaron a cabo intervenciones focalizadas en la modificación del mismo (Mendes de Leon, Powell y Kaplan, 1991). Esto permitió comprender la importancia de factores estructurales como vulnerabilidad para el desarrollo de enfermedades crónicas, a pesar de que actualmente este patrón de personalidad ha perdido relevancia al evidenciarse que la hostilidad era un componente que lo caracterizaba, factor de riesgo presente en esta personalidad que explicaría su asociación con la enfermedad cardiovascular (Juárez-García, 2008; Myrtek, 2001).

Finalmente, como es sabido, el hecho de ser diagnosticado con una enfermedad crónica lleva un proceso de ajuste por el impacto social, emocional y físico en la vida de las personas; sin embargo, para algunos, el ajuste es positivo frente a las enfermedades crónicas (de Ridder, Geenen, Kuijer y van Middendorp, 2008; Helgeson y Zajdel, 2017). Esto señala la necesidad de estudiar los indicadores de un mayor o menor ajuste frente al diagnóstico y vivencia de este tipo de enfermedades, donde la personalidad podría ser importante (Stanton, Revenson y Tennen, 2007).

Cabe anotar que todas las alusiones a la personalidad en la psicología de la salud hacen referencia a ciertos factores, que se han estudiado de forma disgregada o pertenecen a modelos teóricos diferentes. Pocas veces en estos estudios se hace referencia a los patrones psicopatológicos, representados actualmente en el modelo actual asumido por el manual diagnóstico y estadístico de los trastornos mentales en su quinta versión (DSM-5) (American-Psychiatric-Association [APA], 2013), pero mucho más cercano a la propuesta encontrada en su apéndice y a la postura del manual de la CIE en su versión 11 (Figueroa-Cave, 2018). Lo anterior llevó a plantear esta reflexión acerca de los modelos dimensionales de la personalidad y su importancia en el campo de la psicología de la salud, ejemplificándolo en el área de la salud cardiovascular.

\section{De modelos categoriales a dimensionales de la personalidad: los estudios sobre el temperamento}

Los modelos categoriales para el entendimiento de la personalidad no son nuevos. Los cambios en los manuales diagnósticos así lo reflejan, pasando de las 
estructuras específicas (presentes hasta la tercera versión del manual americano) hacia definiciones más amplias de patrones perdurables de experiencia interna y de comportamientos que se desvían notablemente de las expectativas de la cultura del individuo y se expresan en las cogniciones, la afectividad, el funcionamiento interpersonal y el control de los impulsos (APA, 2013). No obstante, estos patrones de personalidad o específicamente, trastornos, terminan llevando a una clasificación nuevamente categorial y por clusters, que no siempre se ajusta a lo que observan los terapeutas a nivel clínico (David y Freeman, 2015) y que no permite comprender las relaciones dosis respuesta de riesgo de padecer cierta enfermedad, ya que más que la presencia de un rasgo de personalidad o la manifestación de una emoción, la relación parece darse con respecto a la frecuencia e intensidad de emociones específicas, las cuales podrían estar asociadas con la tendencia estructural a percibir situaciones de una manera determinada y, desde allí, a una mayor frecuencia de presentación de cierta emoción (Kubzansky, Martin y Buka, 2009; Kubzansky y Thurston, 2007).

Los modelos dimensionales han cobrado aún más importancia gracias a los estudios acerca del temperamento que evidencian que existirían tres dimensiones innatas biocomportamentales; dos de ellas se refieren a la estructura afectiva, afectividad positiva y negativa, y la tercera tendría que ver con la desinhibición o inhibición del sistema nervioso (Clark, 2005). La afectividad positiva y negativa actúan como sistemas de aproximación y retiro respectivamente, mientras que la tercera dimensión regula el grado en que los estímulos entrantes pueden ser inhibidos (Clark, 2005).

De forma semejante, otros estudios sobre el temperamento han señalado que existirían sistemas biocomportamentales más complejos y amplios que los anteriores: el sistema comportamental inhibitorio y el de activación. Estas dimensiones representan diferencias individuales en la sensibilidad de dos sistemas neurológicos. Uno de los sistemas regula la motivación aversiva (sistema de inhibición comportamental [sIC]) y el otro la motivación apetitiva (sistema de activación comportamental [SAC]) (Carver y White, 1994; Watson, 2000).

El SAC ayuda a identificar los estímulos y claves asociadas al reforzamiento positivo, así como a la ausencia del castigo, relacionándose con el afecto positivo. Por otra parte, el sic ayuda a identificar los estímulos asociados con 
el castigo o la aparición de eventos negativos, relacionándose con el afecto negativo o con comportamientos de evitación pasiva, rumiación, que podrían llevar a cuadros tales como la ansiedad (Corr, 2004; Corr y Cooper, 2016; Merchán-Clavellino, Alameda-Bailén, Zayas García y Guil, 2019). Estudios recientes se han enfocado en el estudio del SAC identificando que su estructura factorial podría reflejar un sistema multifacético que se asociaría con las dimensiones del afecto positivo (Dornbach-Bender et al., 2020), mientras que otros estudios señalan que se trataría de un factor general (Kelley et al., 2019).

Los estudios sobre el temperamento han influenciado de forma importante a los modelos dimensionales sobre la personalidad, evidenciando, en algunos casos, relaciones que dan mayor sustento empírico a los modelos propuestos, $\mathrm{y}$ en otros, denotando cómo la personalidad se formaría a partir de estos rasgos temperamentales e influiría en la vulnerabilidad a presentar trastornos mentales, así como en enfermedades crónicas en las cuales los factores emocionales han venido siendo reconocidos como factores de riesgo para su incidencia y mal pronóstico — tal es el caso de las enfermedades cardiovasculares (ECV) (Ito et al., 2019; Jani y Hajloo, 2019; Khosravani, Baseri et al., 2020; Kim, 2020; Sánchez-Rodríguez et al., 2020).

Modelos dimensionales y su aplicación en la psicología de la salud: relaciones con la enfermedad cardiovascular

Modelo de Afectividad Negativa y Positiva. El Modelo de Afectividad Positiva y Negativa se desarrolló inicialmente para explicar los aspectos comunes entre la ansiedad y la depresión (Simms, Grös, Watson y O’Hara, 2008; Watson, Clark y Carey, 1988; Watson, Clark y Tellegen, 1988), modificado posteriormente por Clark y Watson (1991) para dar cuenta del elemento prototípico de la ansiedad. El afecto positivo y el negativo son dos constructos diferenciales importantes para comprender la emoción desde el punto de vista biológico y psicológico (Clark y Watson, 1991). La dimensión del afecto negativo hace referencia a un malestar subjetivo compuesto por varios estados emocionales negativos, como el miedo, tristeza, ira, disgusto y culpa. De forma similar, la dimensión del afecto positivo refleja la co-ocurrencia de estados emocionales 
positivos, llevando a que la persona se sienta entusiasmada, activa y alerta (Watson y Clark, 1992; Watson, Clark y Stasik, 2011; Watson, Clark y Tellegen, 1988; Watson y Pennebaker, 1989).

En ese sentido, se ha pensado que las dimensiones del afecto positivo y negativo son estables y predictivas en el tiempo. Su estabilidad se comprobó en un estudio de seguimiento por 7.5 ańos con estudiantes universitarios (Watson y Walker, 1996). Desde el punto de vista psicopatológico se ha afirmado que la diferenciación entre afecto y afectividad, señalando el estado y el rasgo, podrían dar un panorama más completo de la psicopatología (Clark, 2005). Así, los estados de afecto positivo y negativo están relacionados con rasgos de afectividad positiva y negativa, que reflejan las diferencias individuales en reactividad emocional (Clark y Watson, 1988).

Una ventaja de la utilización de este modelo es que responde a los modelos temperamentales. $\mathrm{Al}$ respecto, la asociación entre el afecto negativo y el sIC se ha verificado en estudios experimentales con sujetos sanos; sin embargo, la asociación entre el afecto positivo y el sAC parece ser más compleja, ya que se han identificado diferentes facetas (Corr, 2004; Dornbach-Bender et al., 2020). Un estudio sobre el volumen de materia gris y cada uno de los sistemas comportamentales dirigido por Barros-Loscertales et al. (2006) encontró que mayores puntuaciones en sic correlacionan con un mayor volumen de la amígdala (núcleo basolateral) y zonas ventrales de la formación hipocampal a nivel cerebral. Estas zonas han sido relacionadas con la sensación de miedo y ansiedad mediante estudios de resonancia magnética funcional que señalan su activación cuando se procesan estímulos aversivos en pacientes con trastornos de ansiedad y personalidades ansiosas (Barros-Loscertales et al., 2006). Así mismo, en un estudio llevado a cabo en Irán con estudiantes universitarios, se encontró que estos sistemas, al igual que la autoestima, están asociados con un $20 \%$ de la varianza de ansiedad social y con un $47 \%$ de la depresión en un estudio llevado a cabo en Irán con estudiantes universitarios (Heidari y Nemattavousi, 2020).

En los estudios en salud se ha encontrado una correlación entre la inhibición y activación comportamental, con mayor riesgo de incidencia cardiovascular y regulación cardíaca (Hintsanen et al., 2009). Al respecto, en una 
muestra de 296 pacientes que habían tenido un síndrome cardiaco agudo (scA) se encontró que la búsqueda de novedad, relacionada con el sAC fue asociada con mejor pronóstico; sin embargo, al ajustar por depresión y anhedonia los efectos dejaron de ser significativos (Leroy, Loas y Perez-Diaz, 2011). Esto puede señalar la alta relación entre tales constructos y la especificidad del afecto negativo y positivo como los elementos específicos relacionados con la ECV.

Personalidad Tipo D. La personalidad tipo D hace referencia a la tendencia a experimentar emociones negativas y a inhibir su expresión en las interacciones sociales (Denollet, Pedersen, Vrints y Conraads, 2006; Denollet et al., 1996; Kupper y Denollet, 2007), al igual que suprimir, reprimir y contener el malestar emocional (Laham, 2008). Así, estas personas experimentarían niveles más crónicos de desasosiego general no detectables, porque el sujeto no los expresa (Smith y Blumenthal, 2011). De este modo, la supresión emocional constituiría el elemento clave de esta personalidad al afrontar su malestar emocional mediante la supresión del malestar (Kupper y Denollet, 2007). Se ha estimado que la prevalencia de la personalidad tipo D estaría entre $24 \%$ y $31 \%$ en la población general y entre $15 \%$ y $53 \%$ en estudios con pacientes con ECV y pacientes con hipertensión arterial; sin embargo, los estudios en los que se basan estos datos no son de muestras poblacionales (Beutel et al., 2012; Compare et al., 2013; Kupper y Denollet, 2007).

Desde los noventa, Denollet et al. (1996) han propuesto que la personalidad tipo D se asociaría con patologías coronarias y ha sido descrito como un predictor independiente de eventos cardíacos adversos en pacientes con ECV, asociándose a casi el doble en riesgo de nuevos eventos cardíacos (Compare et al., 2013; Denollet et al., 2006; Denollet et al., 1996). Al diferenciar entre eventos tempranos (en los primeros seis meses posteriores al IAM) y tardíos (posterior a los primeros seis meses) los resultados de un estudio con 473 pacientes seguidos por 1.8 años muestran que esta personalidad se asociaba con el doble de riesgo de eventos cardíacos tempranos y tres veces el riesgo de eventos tardíos después de ajustar por depresión y severidad de la enfermedad cardíaca (Martens, Mols, Burg y Denollet, 2010). Estos resultados fueron confirmados por una revisión teórica sobre los rasgos de personalidad y sus 
impactos en la mortalidad cardíaca, que sintetizó los resultados de los estudios prospectivos con pacientes con ECV publicados hasta septiembre del 2009 y concluyó que la personalidad tipo D predecía significativamente un peor desempeño físico en todos los grupos evaluados (Reich y Schatzberg, 2010).

Según se ha escrito, la personalidad tipo D afectaría la salud mediante tres trayectorias: primero, incrementaría el riesgo de padecer ansiedad o depresión, factores de riesgo en personas en ECv; segundo, llevaría a una pobre adherencia al tratamiento y a estilos de vida poco saludables; y, tercero, podría asociarse con mecanismos biológicos subyacentes como la inflamación y el desbalance autonómico (Beutel et al., 2012; Kupper y Denollet, 2007; Reich y Schatzberg, 2010). Es importante señalar que no todas las trayectorias han recibido evidencia empírica, por ejemplo, un análisis con población general alemana no encontró asociaciones entre marcadores fisiológicos o con hábitos de vida saludables y la personalidad tipo D; sin embargo, encontró una asociación entre personalidad tipo D, ansiedad y depresión, en donde el $60 \%$ de las personas con depresión y el $54 \%$ de las que presentaron ansiedad cumplieron también los criterios para este tipo de personalidad (Beutel et al., 2012).

Modelo de los cinco factores. Este modelo tiene sus raíces en el paradigma léxico recogiendo lo que es más significativo cuando se describe a una persona y que queda codificado en el lenguaje. A partir de esto se habla de cinco factores, o dominios, en tanto se plantea que dentro de estos existen grados o variaciones que emergen en la medida que las personas desarrollan más palabras para evidenciar los grados o variaciones de cada uno de los factores iniciales (Widiger y Crego, 2019). Es un modelo que ha cobrado importancia en las últimas décadas, sintetizando los rasgos que habían sido identificados previamente en el lenguaje coloquial y en diversas pruebas psicológicas. Los cinco factores que reúne este modelo son neuroticismo, extraversión, apertura a la experiencia, amabilidad y consciencialresponsabilidad (Costa Jr y McCrae, 2008), y cada dimensión tiene una polaridad inversa, por ejemplo neuroticismo - estabilidad emocional (Figueroa-Cave, 2018). Este modelo cuenta con amplia validación empírica, incluyendo estudios de replicación cultural, de genética comportamental, antecedentes en la infancia, estabilidad, neurociencia, y validaciones en diferentes idiomas (Widiger y Crego, 2019). 
El neuroticismo hace referencia a una tendencia relativamente estable de responder con emociones negativas a situaciones de frustración, pérdida o amenaza. En este sentido, las personas que puntúan alto en este rasgo reaccionan con emociones intensas frente a pequeños desafíos (Lahey, 2009). Este factor se ha asociado con menores niveles de calidad de vida en la comunidad en general, ya que incluye la sensación de sentirse enfermo, excesivamente preocupado, fracasado laboralmente y con poca satisfacción marital (Widiger y Oltmanns, 2017); así mismo, este rasgo se ha asociado con una mayor preocupación por la salud, pero menores conductas de protección, lo que recientemente ha llevado a plantear que podría llevar a que aquellos con niveles altos en este rasgo tengan mayor riesgo de ser infectados con Covid-19 al interpretarse en el contexto actual (Khosravi, 2020). Por esta razón, por su correlación con trastornos físicos y emocionales y por el hecho de que las personas con altas puntuaciones en neuroticismo presentan mayor frecuencia de uso de los servicios de salud física y mental, este rasgo es altamente significativo en la salud pública (Lahey, 2009).

La extraversión hace referencia a una tendencia a la sociabilidad, a la asertividad y se expresa en el comportamiento de conversar fácilmente (Costa Jr y McCrae, 2008). Las personas con altos niveles de extraversión reportan tener mayores emociones positivas en el contacto social (Pang et al., 2016), así como mayor afecto positivo, lo cual repercute en la frecuencia, intensidad y duración de las emociones asociadas (Verduyn y Brans, 2012). La apertura a la experiencia se describe como la tendencia a ser de mente abierta y a sentir gusto al experimentar. Las personas con altas puntuaciones en este rasgo son abiertas al mundo y a buscar ideas y emociones nuevas (Costa Jr y McCrae, 2008). Por esto se ha reportado que la apertura se asocia con la capacidad de hacer una revaloración de situaciones inicialmente percibidas como amenazantes (Schneider, Rench, Lyons y Riffle, 2012). La relación entre la apertura a la experiencia y la salud se daría mediante una mayor voluntad para involucrarse en nuevas aproximaciones para el cuidado de la salud o mediante compromisos con dietas más saludables (Koots-Ausmees et al., 2016).

Respecto a la amabilidad, las personas con altas puntuaciones en este factor tienden a ser altruistas y ayudan en todo lo necesario (Costa Jr y McCrae, 
2008). Es importante anotar que una mayor amabilidad se ha asociado con mayor reactividad en la respuesta de experiencias estresantes, pero, al mismo tiempo, los pacientes con altas puntuaciones en amabilidad presentan mayores tendencias a efectuar comportamientos relacionados con la salud, como la notable adherencia a los tratamientos con antibióticos (Koots-Ausmees et al., 2016). Finalmente, la responsabilidad alta se evidenciaría en la tendencia de una persona a ser minuciosa, controlada respecto a sus impulsos, fiable y escrupulosa (Costa Jr y McCrae, 2008). Este rasgo se ha asociado directamente con la probabilidad de inhibir conductas impulsivas que pueden atentar contra la salud; de este modo, personas con bajas puntuaciones en responsabilidad parecen tener más comportamientos de riesgo para la salud como fumar, comer poco saludable, conducir de forma irresponsable y tener intercambios sexuales sin protección (Koots-Ausmees et al., 2016).

Con respecto a la estabilidad de los rasgos, Mroczek y Spiro (2003) encontraron que, aunque se trata de rasgos estables, estos pueden presentar algunas variaciones con la edad. El estudio indicó que existen diferencias estadísticamente significativas en el nivel inicial y en la tasa de cambio de neuroticismo y extraversión en un período de seguimiento de 12 ańos. Adicionalmente, se encontró que los eventos vitales afectan principalmente la tasa de cambio del neuroticismo, por encima de la extraversión y se encontró que el neuroticismo disminuiría con la edad, mientras que la extraversión aumentaría (Mroczek y Spiro, 2003).

Con base en lo anterior es que diferentes investigadores afirman que algunos de estos factores parecen tener mayor implicación en la salud mental y física de las personas y que parecen generar influencias moduladoras. Respecto al neuroticismo y la extraversión, se ha afirmado que estos dos rasgos podrían predecir la variabilidad afectiva, donde el neuroticismo está principalmente relacionado con la intensidad media del afecto negativo; y la extraversión la intensidad media del afecto positivo (Dauvier et al., 2019; Verduyn y Brans, 2012). Adicionalmente, la conjunción entre alto neuroticismo y baja extraversión podría llevar a mayor inhibición comportamental, lo cual se ha relacionado con presentación de mayores síntomas de ansiedad (Klinger-König et al., 2018). 
Con respecto a la ECV, se ha encontrado que la tasa de cambio del estado de salud se vio significativamente afectada por las puntuaciones en neuroticismo. Específicamente, se reportó que por cada punto adicional que una persona tenía en neuroticismo, la probabilidad de morir se incrementaba en un $40 \%$. Este riesgo fue aún mayor en los casos en que altas puntuaciones de neuroticismo se reportaban concomitantemente con otros factores de riesgo para las ECV (Mroczek y Spiro, 2007).

Tomando como referencia a pacientes con aneurismas intracraneales no rotas, en las que debido a las características del aneurisma se había optado por un tratamiento conservador, investigaciones han reportado niveles elevados de responsabilidad, amabilidad y neuroticismo en comparación con la población colombiana, así como bajo en extraversión y apertura; adicionalmente, los análisis realizados llevaron a afirmar que la calidad de vida de los pacientes se veía afectada negativamente por los niveles de ansiedad, los cuales estaban asociados con la personalidad del paciente, donde el neuroticismo se asocia directamente con la sintomatología y la extraversión de forma inversa (Lemos et al., 2020). Estos datos son congruentes con estudios previos que habían señalado que el neuroticismo es una de las variables de peor pronóstico para la calidad de vida en pacientes que habían tenido una hemorragia por ruptura de una aneurisma (Passier et al., 2013), señalando la importancia de la personalidad en personas que presentan una aneurisma, presente ruptura o no. Finalmente, el neuroticismo también se ha asociado a otros problemas salud física tales como eczema atópico, asma, y colon irritable, aun cuando se controla por depresión y otros factores de riesgo (Lahey, 2009).

Otros rasgos de personalidad con influencia en la salud. Uno de los rasgos independientes de la personalidad que ha recibido mayor evidencia es el $o p$ timismo disposicional, entendido como la tendencia a creer que los eventos presentes o futuros tendrán desenlaces favorables (Scheier y Carver, 1985; Scheier, Carver y Bridges, 1994). Se ha encontrado que este rasgo se asocia con un menor riesgo de eventos cardíacos en población con antecedentes cardiovasculares (Bedi y Brown, 2005), así como en muestras epidemiológicas generales (Kubzansky, Sparrow, Vokonas y Kawachi, 2001). Desde la psicología positiva se afirma que el optimismo sería un mediador del proceso de 
valoración del estímulo en el proceso de estrés (Moore, 2002). Al respecto, un metaanálisis de 84 estudios sobre la asociación entre optimismo y salud general encontró que la relación era estadísticamente significativa $(\mathrm{HR}=0.17)$; sin embargo, la asociación fue mayor con salud subjetiva, donde se incluyó la calidad de vida relacionada con la salud, que con la objetiva (Rasmussen, Scheier y Greenhouse, 2009).

En pacientes con ECV se ha reportado que el optimismo predice una mejor recuperación después de una cirugía de bypass en tanto se relaciona con la modificación exitosa de los comportamientos de riesgo y con mayor bienestar emocional después de un evento cardíaco (Bedi y Brown, 2005; Contrada y Goyal, 2004; Fitzgerald, Prochaska y Pransky, 2000). Además, el optimismo en este tipo de pacientes predijo menor recurrencia de eventos cardíacos al finalizar un programa de rehabilitación cardíaca; para este caso, en 164 pacientes seguidos por 12 meses (Hevey, McGee y Horgan, 2014). Respecto a la relación con otros constructos, un seguimiento de ocho meses a una muestra de 284 pacientes depresivos y 146 no depresivos sometidos a una revascularización carotídea encontró que el optimismo predijo índices más elevados de recuperación en los pacientes depresivos a nivel de su CVRs, así como el número de rehospitalizaciones (Tindle et al., 2012).

El optimismo tendría un impacto en la salud cardiovascular mediante mecanismos fisiológicos y comportamentales, sin embargo, la mayoría de los estudios se han adelantado con muestras de personas sanas y no cardiovasculares (Boehm et al., 2020; Rozanski, Bavishi, Kubzansky y Cohen, 2019). Este podría servir como un precursor de comportamientos saludables mediante la motivación para comportarse en formas consistentes con sus expectativas favorables del futuro (Kubzansky, Kubzansky y Maselko, 2004). Además, se ha encontrado que actitudes intrínsecas a este rasgo independiente se asocian con mayores niveles de colesterol HDL y con niveles bajos de triglicéridos (Boe$\mathrm{hm}$ et al., 2013). A nivel comportamental se ha sugerido que tal rasgo puede permitir a los individuos movilizar estrategias de afrontamiento adaptativas como la adopción de conductas saludables y la evitación de conductas menos saludables como el consumo de licor (Fitzgerald et al., 2000; Kubzansky et al., 2004; Kubzansky et al., 2001). Sin embargo, hay evidencia contradictoria o 
no concluyente; por ejemplo, el optimismo se ha asociado también con mayor probabilidad de fumar y menor probabilidad de visitar a un doctor (Kubzansky et al., 2004). Igualmente, en una evaluación de 46 pacientes comenzando un programa de rehabilitación cardíaca el optimismo no se asoció con medidas de resultado de un programa de rehabilitación cardíaca 12 semanas después (Glazer, Emery, Frid y Banyasz, 2002).

Otro rasgo de personalidad que ha mostrado evidencia empírica suficiente en su relación con la salud cardiovascular es la hostilidad, la cual se define como una actitud cognitiva relativamente estable en el tiempo desarrollada por la exposición repetida a situaciones de ira y caracterizada por cinismo, suspicacia y resentimiento hacia los otros, llevando a intercambios negativos y mayores oportunidades de experimentar ira (Kubzansky, Davidson y Rozanski, 2005; Oblitas, 2007; Smith y Ruiz, 2002). Este rasgo se basa en un estilo atribucional explicado por la cognición de amenaza y la presunción de malevolencia por parte de los demás. Consecuentemente las personas hostiles tienden a esperar lo peor de los demás y generalmente están a la defensiva (Kendall-Tackett, 2009; Palmero et al., 2007).

Al respecto, un metaanálisis mostró que la ira/hostilidad incrementa el riesgo de sufrir un incidente cardiovascular y usualmente implica un mal pronóstico tanto en las personas que ya padecían la enfermedad ( $\mathrm{HR}=1.19$; I.C. $95 \%=1.05-1.35)$ como en poblaciones sanas ( $\mathrm{HR}=1.23$; I.C. $95 \%=1.08$ - 1.42). El estudio concluyó que niveles superiores de ira-hostilidad se asocian a evoluciones clínicas de inferior calidad y a incrementos en el número de desenlaces en pacientes con ECv (Chida y Steptoe, 2009). En una línea cercana, Olson et al. (2005) llevaron a cabo un estudio con una cohorte de mujeres con sospecha de presentar un SCA y reportaron datos de 954 mujeres donde los niveles de hostilidad estaban inversamente relacionados con una supervivencia a seis años libre de eventos cardiovasculares. Finalmente, en un estudio de Ochoa et al. (2010) que incluyó a 90 personas con diagnóstico de cardiopatía isquémica y 78 controles de la comunidad general de Medellín se encontraron mayores niveles de ira-rasgo y de la expresión interna de la ira en los participantes pacientes que en los controles (controlando por la edad y el sexo) (Ochoa et al., 2010). 
Cabe anotar que la evidencia también señala otros rasgos de personalidad como importantes en otras enfermedades, como características evitativas y con necesidad de control, asociados con mayor riesgo de fibromialgia (Sayar, Gulec, Topbas y Kalyoncu, 2004).

\section{Discusión y conclusiones}

El recuento llevado a cabo permite evidenciar la importancia que han tenido modelos dimensionales para el entendimiento de los factores de personalidad en la salud. Adicionalmente, señala el interés en el rol de la personalidad en la salud y la enfermedad y la importancia que pueden tener factores de la personalidad en la presentación y cronificación de ciertos cuadros (Denollet, 2000). Estos modelos, cada vez más cercanos a teorías biológicas temperamentales, solían tener más aceptación en otros campos de la psicología, como en clínica y organizacional, como es el caso de los modelos de afectividad negativa y positiva o el modelo de los cinco factores. Ahora, el punto central frente a los modelos dimensionales es determinar qué modelo podría ser el más comprehensivo y explicativo frente a los diferentes fenómenos de la psicopatología y la vulnerabilidad a enfermedades crónicas como las aquí analizadas. Para esto, se procederá a considerar algunos aspectos importantes de los modelos presentados anteriormente.

Respecto a la personalidad tipo D actualmente no hay un consenso frente a su importancia en pacientes con ECV (Coyne y de Voogd, 2012; Lichtman et al., 2014). Se ha señalado que este concepto implica la presencia de dos factores que podrían estar relacionados con la ECV de forma independiente y que su combinación no puede definir por sí misma un tipo de personalidad (Laham, 2008; O’Riordan, Howard, Brown y Gallagher, 2020). Adicionalmente, el hecho de incluir dos elementos que estén correlacionados aumenta la posibilidad de encontrar resultados por azar, de tal forma que algunos autores afirman que su efecto en las investigaciones puede deberse a la suma de efectos individuales (Compare et al., 2013; Lodder, 2020; Lodder, Emons, Denollet y Wicherts, 2020). Sin embargo, Denollet (2000) afirma que, aunque estos constructos están correlacionados, no son idénticos en tanto que el valor del rasgo de inhi- 
bición social a la dimensión del afecto negativo es la noción de que la manera de afrontar las emociones es tan importante como la experimentación de las emociones por sí misma.

Otras críticas a este tipo de personalidad han señalado que enmascara el efecto de la depresión en la ECV y que no se trata realmente de un tipo de personalidad estable tal y como ha sido definido (Lichtman et al., 2014). Esto podría tener relación con datos que indican que las puntuaciones de personalidad tipo D podrían cambiar en mediciones previas y posteriores a un evento cardiaco, lo que no indicaría que se trate de un tipo de personalidad (Beutel et al., 2012). Otra crítica aún más importante es que se ha señalado que casi todos los estudios que evidencian la importancia de la personalidad tipo D como factor pronóstico de mortalidad han sido realizados por el mismo grupo de investigación en Tilburg University, y que en estos se ha presentado un número de muertes a explicar inferior que al que podría ser esperado debido a los factores de riesgo tradicionales para las ECv. Esto resulta en que la reclasificación, alusión o sustracción de una muerte lleven a que los resultados difieran de lo encontrado. Además, en estos estudios hay una variación de los tiempos de seguimiento, de la variable predictora analizada (muertes cardíacas, muertes en general o eventos cardíacos), de la elección de covariables distintas según el artículo y presenta reporte sólo de los resultados ajustados, mas no de resultados sin ajustar (Coyne y de Voogd, 2012).

Finalmente debe señalarse que dentro de esta personalidad se habla del componente de afectividad negativa, que podría considerarse parte de otro modelo y un factor independiente de vulnerabilidad cardiovascular, pero, además, que en ocasiones este concepto se equipara con neuroticismo y posteriormente se establecen diferencias con él (Kupper y Denollet, 2007). No obstante, otras veces se asume la definición de Watson, Clark y Tellegen (1988), pero se utiliza la escala de ansiedad rasgo para medirlo (Denollet y de Potter, 1992; Denollet et al., 1996) así como ítems del MMPI (Kupper y Denollet, 2007). Este último punto posiblemente señale una falla conceptual importante frente a la personalidad tipo D y evidencia el alto solapamiento entre modelos, donde el rasgo que parece ser común a todos es la afectividad negativa o neuroticismo, según como sea definido. 
Al respecto de la afectividad negativa cabe aclarar que desde sus primeras definiciones conceptuales se correlacionó con el neuroticismo, mientras que la afectividad positiva con la extraversión (Watson, Clark y Tellegen, 1988). Esto se ha evidenciado empíricamente en estudios con estudiantes universitarios, en los que se encontró que aquellos con alto afecto negativo y bajo afecto positivo mostraron mayores niveles en neuroticismo, vulnerabilidad, ansiedad, depresión y rumiación, así como menores niveles en satisfacción con la vida (Zanon y Hutz, 2013). De forma semejante un análisis secundario de una base de datos de pacientes con trastornos del estado del ánimo y ansiedad evidenció que el neuroticismo podría ser un factor diferencial entre aquellos pacientes que sólo presentan un trastorno y los que presentan varios; esto arroja evidencia sobre el neuroticismo como factor de vulnerabilidad para los trastornos mentales pero también sobre la asociación entre éste y el afecto negativo, el cual se considera el factor latente tras la comorbilidad de los trastornos (Cuijpers, van Straten y Donker, 2005). Finalmente, se resalta que los autores del modelo argumentan que el neuroticismo, como rasgo de personalidad, refiere a una dimensión amplia referida a la probabilidad de experimentar emociones negativas; no obstante, la experiencia emocional estaría dominada por dos dimensiones amplias e independientes, el afecto negativo y el positivo (Watson y Pennebaker, 1989).

Aun así, para algunos investigadores la afectividad negativa no sólo agruparía el factor común entre la ansiedad y la depresión —en este caso, como rasgo-, sino que también incluiría un factor común con el complejo ira-hostilidad. Al respecto, una investigación llevada a cabo en población sin ECV encontró esta relación y la asoció con los niveles de variabilidad cardíaca, evidenciando una relación entre este constructo y una mayor vulnerabilidad a desarrollar patologías cardiovasculares en el futuro (Bleil et al., 2008). Así mismo, en pacientes con ECV colombianos que habían sido hospitalizados por un síndrome coronario agudo también se confirmó que el afecto negativo es el factor de segundo orden entre la subescala de afecto negativo de la ansiedad rasgo, la subescala de temperamento de la ira rasgo y los dos componentes de la prueba de depresión rasgo (Lemos y Agudelo-Vélez, 2018).

Frente al modelo de los cinco factores, se ha afirmado que es el modelo que viene teniendo mayor reconocimiento por ser comprehensivo y tener 
mayor evidencia empírica (David y Freeman, 2015). Así mismo, que este modelo parece haber sido una de las bases para el establecimiento de los rasgos con los que se definen los trastornos de la personalidad desde los modelos dimensionales en los manuales diagnósticos (Figueroa-Cave, 2018; Widiger y Crego, 2019).

No obstante, sobresale el hecho de que, aunque algunos estudios señalan trayectorias muy claras entre el neuroticismo y ciertas patologías como las ECV, estas medidas no son independientes de las de depresión y ansiedad (Lahey, 2009), de tal forma que la interpretación de las correlaciones debe ser cautelosa y es importante considerar el uso de modelos estructurales que permitan comprender el efecto de varias variables sobre la patología, para calcular el peso específico de cada una de ellas.

Para finalizar, es importante considerar aquellas trayectorias que parecen ser comunes a los modelos propuestos frente a la influencia de la personalidad en la salud. Es posible que los rasgos de personalidad afecten los resultados médicos de varias maneras, de forma directa o indirecta. De forma directa podría pensarse que rasgos como el neuroticismo estarían asociados con factores genéticos que llevarían a una mayor reactividad del eje hipotálamo-pituitario-adrenal y con mayores alteraciones del sistema inmune en respuesta a eventos vitales estresantes. Estas respuestas fisiológicas podrían contribuir a mayores problemas físicos.

Igualmente, los rasgos elevados de personalidad están asociados a una mayor probabilidad de presentar ansiedad o depresión; de esta forma, podrían ser las emociones las que afectan la condición física, especialmente en los casos que se vuelven crónicas o resistentes. El estrés ocasionado por estos trastornos o los tratamientos que se utilizan para los mismos podrían también tener efectos en los resultados físicos. Ahora, con respecto a patrones de actuación comportamental, se ha afirmado que los rasgos de personalidad interfieren con el acceso o la habilidad a permanecer en un tratamiento (Reich y Schatzberg, 2010) y que las personas con ciertos rasgos podrían engancharse más fácilmente en comportamientos que aumentan el riesgo de problemas físicos; por ejemplo, fumar, presentar dependencia al alcohol u a otras sustancias (Lahey, 2009). 
Empero, los rasgos de personalidad no son necesariamente inmodificables e inflexibles. Existen componentes que son modificables y se ha encontrado que estos cambios pueden tener efectos significativos en la mortalidad. Si esto es así, podría haber intervenciones que movilicen los rasgos en una dirección que lleve a una disminución de la mortalidad (Reich y Schatzberg, 2010).

Para concluir, se señala la importancia de reconocer que los modelos de vulnerabilidad-estrés permiten comprender mejor el desarrollo de patologías, no sólo mentales sino también físicas y que, en estos modelos, la presencia de rasgos de personalidad viene señalándose como un factor importante. Varios de los modelos aquí descritos tienen fortalezas, mientras que algunos están siendo sometidos a críticas que llevan a dudar de su validez conceptual. Es importante tener presente estos puntos al momento de considerar la evaluación de las variables de la personalidad en un estudio en el campo de la psicología de la salud.

\section{Referencias}

American-Psychiatric-Association. (2013). Diagnostic and Statistical Manual of Mental Disorders, Fifth Edition (DSM 5). Arlington, VA: American Psychiatric Association.

Amodio, D., Master, S., Yee, C. y Taylor, S. (2008). Neurocognitive Components of the Behavioral Inhibition and Activation Systems: Implications for Theories of Self-Regulation. Psychophysiology, 45(1), 11-19. doi: 10.1111/j.14698986.2007.00609.x.

Barros-Loscertales, A., Meseguer, V., Sanjuan, A., Belloch, V., Parcet, M., Torrubia, R. y Avila, C. (2006). Behavioral Inhibition System Activity is Associated with Increased Amygdala and Hippocampal Gray Matter Volume: A Voxel-Based Morphometry Study. Neuroimage, 33(3), 1011-1015. doi: 10.1016/j.neuroimage.2006.07.025.

Bedi, G. y Brown, S. (2005). Optimism, Coping Style and Emotional Well-Being in Cardiac Patients. British journal of health psychology, 10(1), 57-70. doi: 10.1348/135910704X15266.

Beutel, M., Wiltink, J., Till, Y., Wild, P., Münzel, T., Ojeda, F., Zeller, T., Schnabel, R., Lackner, K., Blettner, M., Zwiener, I. y Michal, M. (2012). Type D Perso- 
nality as a Cardiovascular Risk Marker in the General Population: Results From the Gutenberg Health Study. Psychotherapy and psychosomatics, 81(2), 108117. doi: $10.1159 / 000331776$.

Bleil, M., Gianaros, P., Jennings, J., Flory, J. y Manuck, S. (2008). Trait Negative Affect: Toward an Integrated Model of Understanding Psychological Risk for Impairment in Cardiac Autonomic Function. Psychosomatic Medicine, 70(3), 328-337. doi: 10.1097/psy.0b013e31816baefa.

Boehm, J., Qureshi, F., Chen, Y., Soo, J., Umukoro, P., Hernandez, R., Lloyd-Jones, D. Kubzansky, L. (2020). Optimism and Cardiovascular Health: Longitudinal Findings From the Coronary Artery Risk Development in Young Adults Study. Psychosomatic medicine, 82(8), 774-781. https://doi.org/10.1097/ PSY.0000000000000855

Boehm, J., Williams, D., Rimm, E., Ryff, C. y Kubzansky, L. (2013). Relation Between Optimism and Lipids in Midlife. The American journal of cardiology, 111(10), 1425-1431. doi: 10.1016/j.amjcard.2013.01.292.

Carver, C. y White, T. (1994). Behavioral Inhibition, Behavioral Activation, and Affective Responses to Impending Reward and Punishment: the BIS/BAS Scales. Journal of personality and social psychology, 67(2), 319-333. doi: 10.1037/0022-3514.67.2.319.

Chida, Y. y Steptoe, A. (2009). The Association of Anger and Hostility With Future Coronary Heart Disease: A Meta-analytic Review of Prospective Evidence. Journal of the American College of Cardiology, 53(11), 936-946. doi: 10.1016/j.jacc.2008.11.044.

Chimenti, M., Fonti, G., Conigliaro, P., Hitaj, J., Triggianese, P., Teoli, M., Galluzzo, M., Talamonti, M., Kroegler, B., Greco, E. y Perrricone, R. (2019). Evaluation of Alexithymia in Patients Affected by Rheumatoid Arthritis and Psoriatic Arthritis: A Cross-sectional Study. Medicine, 98(4). doi: 10.1097/ md.0000000000013955

Clark, L. (2005). Temperament as a Unifying Basis for Personality and Psychopathology. Journal of Abnormal Psychology, 114(4), 505-521. doi: 10.1037/0021843X.114.4.505.

Clark, L. y Watson, D. (1988). Mood and the Mundane: Relations Between Daily Life Events and Self-reported Mood. Journal of Personality and Social Psychology, 54(2), 296-308. doi: 10.1037/0022-3514.54.2.296.

Clark, L. y Watson, D. (1991). Tripartite Model of Anxiety and Depression: Psychometric Evidence and Taxonomic Implications. Journal of Abnormal Psychology, 100(3), 316-336. doi: 10.1037/0021-843X.100.3.316. 
Compare, A., Bigi, R., Orrego, P., Proietti, R., Grossi, E. y Steptoe, A. (2013). Type D Personality is Associated With the Development of Stress Cardiomyopathy Following Emotional Triggers. Annals of Behavioral Medicine, 1-9.doi: $10.1007 / \mathrm{s} 12160-013-9474-\mathrm{x}$.

Contrada, R. y Goyal, T. (2004). Individual Differences, Health and Illness: The Role of Emotional Traits and Generalized Expectancies. En: Sutton, A. Baum, y Johnston, M. (Eds.), The SAGE Handbook of Health Psychology (pp. 143-168). Londres: SAGE publications.

Corr, P. (2004). Reinforcement Sensitivity Theory and Personality. Neuroscience \& Biobehavioral Reviews, 28(3), 317-332. doi: 10.1016/j.neubiorev.2004.01.005.

Corr, P. y Cooper, A. (2016). The Reinforcement Sensitivity Theory of Personality Questionnaire (RST-PQ): Development and Validation. Psychological Assessment, 28(11), 1427-1440. doi: 10.1037/pas0000273.

Costa Jr, P. y McCrae, R. (2008). Inventario de personalidad NEO Revisado (NEO PIR). Inventario NEO reducido de cinco factores (NEO-FFI). Manual Profesional (Cordero, Pamos, A, y Seisdedos, N. Trans. 3 ed.). Madrid: TEA ediciones.

Coyne, J. y de Voogd, J. (2012). Are we Witnessing the Decline Effect in the Type D Personality Literature? What can be learned? Journal of Psychosomatic Research, 73(6), 401-407. doi: 10.1016/j.jpsychores.2012.09.016.

Cuijpers, P., van Straten, A. y Donker, M. (2005). Personality Traits of Patients With Mood and Anxiety Disorders. Psychiatry Research, 133(2), 229-237. doi: 10.1016/j.psychres.2004.10.006.

Dauvier, B., Pavani, J., Le Vigouroux, S., Kop, J. y Congard, A. (2019). The Interactive Effect of Neuroticism and Extraversion on the Daily Variability of Affective States. Journal of Research in Personality, 78, 1-15. doi: 10.1016/j. jrp.2018.10.007.

David, D. y Freeman, A. (2015). Overview of Cognitive-Behavioral Therapy of Personality Disorders. En:Beck, A., Davis, D. yFreeman, A. (Eds.), Cognitive Therapy of Personality Disorders (pp. 3-18). New York: The Guilford Press.

de Ridder, D., Geenen, R., Kuijer, R. y van Middendorp, H. (2008). Psychological Adjustment to Chronic Disease. The Lancet, 372(9634), 246-255. doi: 10.1016/S0140-6736(08)61078-8.

Denollet, J. (2000). Type D personality: a potential risk factor refined. Journal of psychosomatic research, 49(4), 255-266. https://doi.org/10.1016/S00223999(00)00177-X 
Denollet, J. y de Potter, B. (1992). Coping Subtypes For Men With Coronary Heart Disease: Relationship to Well-Being, Stress and Type-A Behaviour. Psychological Medicine, 22(03), 667-684. doi: 10.1017/S0033291700038113.

Denollet, J., Pedersen, S., Vrints, C. y Conraads, V. (2006). Usefulness of Type D Personality in Predicting Five-Year Cardiac Events Above and Beyond Concurrent Symptoms of Stress in Patients With Coronary Heart Disease. The American Journal of Cardiology, 97(7), 970-973. doi: 10.1016/j.amjcard.2005.10.035.

Denollet, J., Rombouts, H., Gillebert, T., Brutsaert, D., Sys, S. y Stroobant, N. (1996). Personality as Independent Predictor of Long-Term Mortality in Patients With Coronary Heart Disease. The Lancet, 347(8999), 417-421. doi: 10.1016/S0140-6736(96)90007-0.

Dornbach-Bender, A., Ruggero, C., Smith, P., Schuler, K., Bennett, C., Neumann, C. y Callahan, J. (2020). Association of Behavioral Activation System Sensitivity to Lower Level Facets of Positive Affect in Daily Life. 152, 109570. doi: 10.1016/j.paid.2019.109570.

Everly, G. y Lating, J. (2002). A Clinical Guide to the Treatment of the Human Stress Response (2nd ed.). London.

Figueroa-Cave, G. (2018). Una nueva propuesta de clasificación de los trastornos de personalidad: la clasificación internacional de enfermedades CIE-11. Revista chilena de neuro-psiquiatría, 56(4), 260-268. doi: 10.4067/s071792272018000400260 .

Fitzgerald, T., Prochaska, J. y Pransky, G. (2000). Health Risk Reduction and Functional Restoration Following Coronary Revascularization: A Prospective Investigation Using Dynamic Stage Typology Clustering. International Journal of Rehabilitation and Health, 5(2), 99-116. doi: 10.1023/A:1012954105557.

Friedman, M. y Roseman, R. (1976). Conducta tipo “A” y su corazón. In: Grijalbo.

García, L., Piqueras, J., Rivero, R., Ramos, V. y Oblitas, L. (2008). Panorama de la psicología clínica y de la salud. Revista CES Psicología, 1(1), 70-93.

Glazer, K., Emery, C., Frid, D. y Banyasz, R. (2002). Psychological Predictors of Adherence and Outcomes Among patients in cardiac rehabilitation. Journal of Cardiopulmonary Rehabilitation and Prevention, 22(1), 40-46. https://doi. org/10.1097/00008483-200201000-00006

Graham-Engeland, J., Song, S., Mathur, A., Wagstaff, D., Klein, L., Whetzel, C. y Ayoub, W. (2018). Emotional State Can Affect Inflammatory Responses to Pain Among Rheumatoid Arthritis Patients: Preliminary Findings. Psychological Reports, 122(6), 2026-2049. doi: 10.1177/0033294118796655. 
Heidari, P. y Nemattavousi, M. (2020). Behavioral Inhibition/Activation Systems and Self-Esteem with Depression: The Mediating Role of Social Anxiety. Journal of Rational-Emotive \& Cognitive-Behavior Therapy, 1-15. doi: 10.1007/ s10942-020-00378-9.

Helgeson, V. y Zajdel, M. (2017). Adjusting to chronic health conditions. Annual reviewofpsychology, 68, 545-571.doi: 10.1146/annurev-psych-010416-044014.

Hevey, D., McGee, H. y Horgan, J. (2014). Comparative Optimism Among Patients With Coronary Heart Disease (CHD) is Associated With Fewer Adverse Clinical Events 12 Months Later. Journal of Behavioral Medicine, 37(2), 300-307. doi: 10.1007/s10865-012-9487-0.

Hintsanen, M., Pulkki-Råback, L., Juonala, M., Viikari, J., Raitakari, O. y Keltikangas-Järvinen, L. (2009). Cloninger's Temperament Traits and Preclinical Atherosclerosis: The Cardiovascular Risk in Young Finns Study. Journal of Psychosomatic Research, 67(1), 77-84. doi: 10.1016/j.jpsychores.2009.01.002.

Islami, F., Goding Sauer, A., Miller, K., Siegel, R., Fedewa, S., Jacobs, E., McCullough, M. Patel, A., Ma, J., Soerjomataram, I., Flanders, D., Brawley, O., Gapstur, S. y Jemal, A. (2018). Proportion and Number of Cancer Cases and Deaths Attributable to Potentially Modifiable Risk Factors in the United States. CA: A Cancer Journal for Clinicians, 68(1), 31-54. doi: 10.3322/caac.21440.

Ito, R., Kobayashi, N., Yokoyama, S., Irino, H., Takebayashi, Y. y Suzuki, S. (2019). Interaction Effects of Behavioral Inhibition System/Behavioral Activation System and Cost/Probability Biases on Social Anxiety. Frontiers in Psychology, 10, 2536. doi: 10.3389/fpsyg.2019.02536.

Jani, S. y Hajloo, N. (2019). The Role of Brain/Behavioral Systems in Prediction of Clinical Psychological Symptoms in Cancer Patients. Iranian Journal of Cancer Care, 1(2), 55-62. doi: 10.29252/ijca.1.2.55.

Juárez-García, A. (2008). La perspectiva multinivel en las enfermedades cardiovasculares: el rol de las características psicosociales del trabajo. Revista Psicología. com, 12(1).

Kazarian, S. y Evans, D. (2001). Health Psychology and Culture: Embracing the 21st Century. En: Kazarin, S. y Evans, D. (Eds.), Handbook of Cultural Psychology (pp. 3 - 24). San Diego, CA: Academic Press.

Kelley, N., Kramer, A., Young, K., Echiverri-Cohen, A., Chat, I., Bookheimer, S., Nusslock, R., Craske, M. y Zinbarg, R. (2019). Evidence For a General Factor of Behavioral Activation System Sensitivity. Journal of Research in Personality, 79, 30-39. doi: 10.1016/j.jrp.2019.01.002. 
Kendall-Tackett, K. (2009). Psychological Trauma and Physical Health: A Psychoneuroimmunology Approach to Etiology of Negative Health Effects and Possible Interventions. Psychological Trauma: Theory, Research, Practice, and Policy, 1(1), 35-48. doi: 10.1037/a0015128.

Khosravani, V., Baseri, A., Kamali, Z., Mohammadzadeh, A. y Amirinezhad, A. (2020). Direct and Indirect Effects of Behavioral Inhibition/Activation Systems on Depression and Current Suicidal Ideation Through Rumination and Self-Reflection. Archives of Suicide Research, 24(4), 568-588. doi: 10.1080/13811118.2019.1649224.

Khosravi, M. (2020). Neuroticism as a Marker of Vulnerability to COVID-19 Infection. Psychiatry Investigation, 17(7), 710. doi: 10.30773/pi.2020.0199.

Kim, B. (2020). Behavioral Activation System (BAS) Dysregulation and Bipolar Spectrum Psychopathology in Daily Life: An Online-Diary Study. 291, 113180. doi: 10.1016/j.psychres.2020.113180.

Klinger-König, J., Hertel, J., Terock, J., Völzke, H., Van der Auwera, S. y Grabe, H. (2018). Predicting Physical and Mental Health Symptoms: Additive and Interactive Effects of Difficulty Identifying Feelings, Neuroticism and Extraversion. Journal of Psychosomatic Research, 115, 14-23. doi: 10.1016/j.jpsychores.2018.10.003.

Koots-Ausmees, L., Schmidt, M., Esko, T., Metspalu, A., Allik, J. y Realo, A. (2016). The Role of the Five-Factor Personality Traits in General Self-Rated Health. European Journal of Personality, 30(5), 492-504. doi: 10.1002/per.2058.

Kubzansky, L., Davidson, K. y Rozanski, A. (2005). The Clinical Impact of Negative Psychological States: Expanding the Spectrum of Risk for Coronary Artery Disease. Psychosomatic Medicine, 67(Supplement 1), S10-S14. doi: 10.1097/ 01.psy.0000164012.88829.41.

Kubzansky, L., Kubzansky, P. y Maselko, J. (2004). Optimism and Pessimism in the Context of Health: Bipolar Opposites or Separate Constructs? Personality and Social Psychology Bulletin, 30(8), 943-956. doi: 10.1177/0146167203262086.

Kubzansky, L., Martin, L. y Buka, S. (2009). Early Manifestations of Personality and Adult Health: A Life Course Perspective. Health Psychology, 28(3), 364-372. doi: $10.1037 / \mathrm{a} 0014428$.

Kubzansky, L., Sparrow, D., Vokonas, P. y Kawachi, I. (2001). Is the Glass Half Empty or Half Full? A Prospective Study of Optimism and Coronary Heart Disease in the Normative Aging Study. Psychosomatic Medicine, 63(6), 910-916. doi: 10.1097/00006842-200111000-00009. 
Kubzansky, L. y Thurston, R. C. (2007). Emotional Vitality and Incident Coronary Heart Disease: Benefits of Healthy Psychological Functioning. Archives of General Psychiatry, 64(12), 1393-1401. doi: 10.1001/archpsyc.64.12.1393.

Kupper, N. y Denollet, J. (2007). Type D Personality as a Prognostic Factor in Heart Disease: Assessment and Mediating Mechanisms. Journal of Personality Assessment, 89(3), 265-276. doi: 10.1080/00223890701629797.

Laham, M. (2008). Psicocardiología: su importancia en la prevención y la rehabilitación coronarias. Suma psicológica, 15(1), 143-170.

Lahey, B. (2009). Public Health Significance of Neuroticism. American Psychologist, 64(4), 241-256. doi: 10.1037/a0015309.

Lazarus, R. (1999). Stress and Emotion. A New Synthesis. New York: Springer.

Lemos, M. y Agudelo-Vélez, D. (2018). El afecto negativo como factor emocional de orden superior en pacientes cardiovasculares. Acta Colombiana de Psicología, 21(2), 68-77. doi: 10.14718/ACP.2018.21.2.4.

Lemos, M., Román-Calderón, J., Calle, G., Gómez-Hoyos, J. y Jimenez, C. (2020). Personality and Anxiety are Related to Health-Related Quality of Life in Unruptured Intracranial Aneurysm Patients Selected for Non-Intervention: A Cross Sectional Study. PLOS ONE, 15(3), e0229795. doi: 10.1371/journal. pone.0229795.

Leroy, M., Loas, G. y Perez-Diaz, F. (2011). Cloninger's Temperament Traits as Predictors of Clinical Events after Acute Coronary Syndrome: A Three-Year Prospective Study. Psychotherapy and Psychosomatics, 80(2), 125-126. doi: $10.1159 / 000319705$.

Lichtman, J., Froelicher, E., Blumenthal, J., Carney, R., Doering, L., Frasure-Smith, N., Freedland, K., Jaffe, A., Leifheit-Limson, E., Sheps, D., Vaccarino, V., Wulsin, L. y American Heart Association Statistics Committee of the Council on Epidemiology and Prevention and Council on Cardiovascular and Stroke Nursing (2014). Depression as a Risk Factor for Poor Prognosis Among Patients With Acute Coronary Syndrome: Systematic Review and Recommendations: A Scientific Statement From the American Heart Association. Circulation. doi: 10.1161/CIR.0000000000000019.

Lodder, P. (2020). A Re-Evaluation of the Type D Personality Effect. 167, 110254. doi: $10.1016 /$ j.paid.2020.110254.

Lodder, P., Emons, W., Denollet, J. y Wicherts, J. (2020). Latent Logistic Interaction Modeling: A Simulation and Empirical Illustration of Type D Personality. 
Los modelos dimensionales de la personalidad y su importancia en la psicología de la salud

Structural Equation Modeling: A Multidisciplinary Journal, 57(11), 1-23. doi: 10.1080/10705511.2020.1838905.

Martens, E., Mols, F., Burg, M. y Denollet, J. (2010). Type D Personality Predicts Clinical Events After Myocardial Infarction, Above and Beyond Disease Severity and Depression. The Journal of Clinical Psychiatry, 71(6), 778-783. doi: $10.4088 /$ jcp.08m04765blu.

Mendes de Leon, C., Powell, L. y Kaplan, B. (1991). Change in Coronary-Prone Behaviors in the Recurrent Coronary Prevention Project. Psychosomatic Medicine, 53(4), 407-419. doi: 10.1097/00006842-199107000-00006.

Merchán-Clavellino, A., Alameda-Bailén, J., Zayas-García, A. y Guil, R. (2019). Mediating Effect of Trait Emotional Intelligence Between the Behavioral Activation System (BAS)/Behavioral Inhibition System (BIS) and Positive and Negative Affect. Frontiers in Psychology, 10(424). doi: 10.3389/fpsyg.2019.00424.

Moore, K. (2002). Positive Psychology and Health: Situational Dependence and Personal Striving. En: Frydenberg, E. (Ed.), Beyond Coping: Meeting Goals, Visions, and Challenges (pp. 107-125). New York: Oxford University Press.

Moser, D., McKinley, S., Riegel, B., Doering, L., Meischke, H., Pelter, M., Davidson, P., Baker, H. y Dracup, K. (2011). Relationship of Persistent Symptoms of Anxiety to Morbidity and Mortality Outcomes in Patients With Coronary Heart Disease. Psychosomatic Medicine, 73(9), 803-809. doi: 10.1097/PSY. 0b013e3182364992.

Mroczek, D. y Spiro, A. (2003). Modeling Intraindividual Change in Personality Traits: Findings From the Normative Aging Study. The Journals of Gerontology Series B: Psychological Sciences and Social Sciences, 58(3), P153-P165. doi: 10.1093/geronb/58.3.P153.

Mroczek, D. y Spiro, A. (2007). Personality Change Influences Mortality in Older Men. Psychological Science, 18(5), 371-376. doi: 10.1111/j.14679280.2007.01907.x.

Myrtek, M. (2001). Meta-analysis of Prospective Studies on Coronary Heart Disease, Type A Personality, and Hostility. International Journal of Cardiology, 79(23), 245-251. doi: 10.1016/S0167-5273(01)00441-7.

Oblitas, L. (2007). Psicología de la Salud y Calidad de Vida. México: Thompson.

Ochoa, A., Herrera, C., Lemos, M., Arango, J. y Rogers, H. (2010). Análisis de la ira en pacientes con cardiopatía isquémica de la ciudad de Medellín (Colombia). Psicología desde el Caribe(26), 86-102. 
Olson, M., Krantz, D., Kelsey, S., Pepine, C., Sopko, G., Handberg, E., Rogers, W., Gierach, G., McClure, C., Merz, C. y WISE Study Group (2005). Hostility Scores Are Associated With Increased Risk of Cardiovascular Events in Women Undergoing Coronary Angiography: A Report from the NHLBI-Sponsored WISE Study. Psychosomatic Medicine, 67(4), 546-552. doi: 10.1097/01. psy.0000170830.99263.4e.

O'Riordan, A., Howard, S., Brown, E. y Gallagher, S. (2020). Type D personality and cardiovascular reactivity to acute stress: The mediating effects of social support and negative social relationships. Psychophysiology, 57(11), e13660. https:// doi.org/10.1111/psyp.13660

Palmero, F., Gómez, C., Guerrero, C., Carpi, A., Díez, J. y Diago, J. (2007). Hostilidad, psicofisiología y salud cardiovascular. Avances en Psicología Latinoamericana, 25(1), 22-43. Recuperado de: http://www.scielo.org.co/pdf/apl/v25n1/ v25n1a4.pdf

Pang, Y., Cui, Q., Wang, Y., Chen, Y., Wang, X., Han, S., Zhang, Z., Lu, G. y Chen, H. (2016). Extraversion and neuroticism related to the resting-state effective connectivity of amygdala. Scientific Reports, 6, 35484. https://doi. org/10.1038/srep35484

Passier, P., Visser-Meily, J., Rinkel, G., Lindeman, E. y Post, M. (2013). Determinants of Health-Related Quality of Life After Aneurysmal Subarachnoid Hemorrhage: A Systematic Review. Quality of Life Research, 22(5), 1027-1043. doi: 10.1007/s11136-012-0236-1.

Rasmussen, H., Scheier, M. y Greenhouse, J. (2009). Optimism and Physical Health: A Meta-analytic Review. Annals of Behavioral Medicine, 37(3), 239-256. doi: 10.1007/s12160-009-9111-x.

Reich, J. y Schatzberg, A. (2010). Personality Traits and Medical Outcome of Cardiac Illness. Journal of Psychiatric Research, 44(15), 1017-1020. doi: 10.1016/j. jpsychires.2010.03.016.

Rice, S., Kealy, D., Ogrodniczuk, J., Seidler, Z., Denehy, L. y Oliffe, J. (2020). The Cost of Bottling It Up: Emotion Suppression as a Mediator in the Relationship Between Anger and Depression Among Men with Prostate Cancer. Cancer management and research, 12, 1039-1046. doi: 10.2147/cmar.s237770.

Rozanski, A., Bavishi, C., Kubzansky, L. y Cohen, R. (2019). Association of Optimism With Cardiovascular Events and All-Cause Mortality: A Systematic Review and Meta-analysis. JAMA Network Open, 2(9), e1912200-e1912200. doi: 10.1001/jamanetworkopen.2019.12200. 
Sayar, K., Gulec, H., Topbas, M. y Kalyoncu, A. (2004). Affective distress and fibromyalgia. Swiss Medical Weekly, 134(17-18), 248-253.

Scheier, M. y Carver, C. (1985). Optimism, Coping, and Health: Assessment and Implications of Generalized Outcome Expectancies. Health psychology, 4(3), 219-doi: 10.1037/0278-6133.4.3.219.

Scheier, M., Carver, C. y Bridges, M. (1994). Distinguishing Optimism From Neuroticism (and Trait Anxiety, Self-Mastery, and Self-Esteem): A Reevaluation of the Life Orientation Test. Journal of Personality and Social Psychology, 67(6), 1063-1078. doi: 10.1037/0022-3514.67.6.1063.

Schneider, T., Rench, T., Lyons, J. y Riffle, R. (2012). The influence of neuroticism, extraversion and openness on stress responses. Stress and Health, 28(2), 102110. https://doi.org/10.1002/smi.1409

Simms, L., Grös, D., Watson, D. y O’Hara, M. (2008). Parsing the General and Specific Components of Depression and Anxiety With Bifactor Modeling. Depression and Anxiety, 25(7), E34-E46. doi: 10.1002/da.20432.

Smith, P. y Blumenthal, J. (2011). Aspectos psiquiátricos y conductuales de la enfermedad cardiovascular: epidemiología, mecanismos y tratamiento. Revista Española de Cardiología, 64(10), 924-933. doi: 10.1016/j.recesp.2011.06.003.

Smith, T. y Ruiz, J. (2002). Psychosocial Influences on the Development and Course of Coronary Heart Disease: Current Status and Implications for Research and Practice. Journal of Consulting and Clinical Psychology, 70(3), 548. doi: 10.1037/0022-006X.70.3.548.

Stanton, A., Revenson, T. y Tennen, H. (2007). Health Psychology: Psychological Adjustment to Chronic Disease. Annual Review of Psychology, 58, 565-592. doi: 10.1146/annurev.psych.58.110405.085615.

Sánchez-Rodríguez, E., Racine, M., Castarlenas, E., Tomé-Pires, C., Galán, S., Jensen, M. y Miró, J. (2020). Behavioral Activation and Inhibition Systems: Further Evaluation of a BIS-BAS Model of Chronic Pain. Pain Medicine. doi: $10.1093 / \mathrm{pm} / \mathrm{pnaa330.}$

Tindle, H., Belnap, B., Hum, B., Houck, P., Mazumdar, S., Scheier, M., Matthews, K., He, F. y Rollman, B. (2012). Optimism, Response to Treatment of Depression, and Rehospitalization After Coronary Artery Bypass Graft Surgery. Psychosomatic Medicine, 74(2), 200-207. doi: 10.1097/PSY.0b013e318244903f.

Verduyn, P. y Brans, K. (2012). The Relationship Between Extraversion, Neuroticism and Aspects of Trait Affect. Personality and Individual Differences, 52(6), 664-669. doi: 10.1016/j.paid.2011.12.017. 
Watson, D. (2000). Mood and Temperament. New York: Guilford Press.

Watson, D. y Clark, L. (1992). Affects Separable and Inseparable: On the Hierarchical Arrangement of the Negative Affects. Journal of Personality and Social Psychology, 62(3), 489 - 505. doi: 10.1037/0022-3514.62.3.489.

Watson, D., Clark, L. y Carey, G. (1988). Positive and Negative Affectivity and Their Relation to Anxiety and Depressive Disorders. Journal of Abnormal Psychology, 97(3), 346. doi: 10.1037/0021-843X.97.3.346.

Watson, D., Clark, L. y Stasik, S. (2011). Emotions and the Emotional Disorders: A Quantitative Hierrarchical Perspective. International Journal of clinical and Health Psychology, 11(3), 429-442.

Watson, D., Clark, L. y Tellegen, A. (1988). Development and Validation of Brief Measures of Positive and Negative Affect: The PANAS Scales. Journal of Personality and Social Psychology, 54(6), 1063-1070. doi: 10.1037/00223514.54.6.1063.

Watson, D. y Pennebaker, J. (1989). Health Complaints, Stress, and Distress: Exploring the Central Role of Negative Affectivity. Psychological Review, 96(2), 234.

Widiger, T. y Crego, C. (2019). The Five Factor Model of Personality Structure: An Update. World Psychiatry, 18(3), 271-272. doi: 10.1002/wps.20658.

Widiger, T. y Oltmanns, J. (2017). Neuroticism is a Fundamental Domain of Personality With Enormous Public Health Implications. World Psychiatry, 16(2), 144-145. doi: 10.1002/wps.20411.

Zanon, C. y Hutz, C. (2013). Affective Disposition, Thinking Styles, Neuroticism and Life Satisfaction. Universitas Psychologica, 12(2), 403-411. doi: 10.11144/ Javeriana.UPSY12-2.adts. 